2. V. S. Rogozin, Two sufficient conditions for the univalence of a mapping, Rostov Gos. Univ. Uč. Zap. Fiz.-Mat. Fak. vol. 32 (1955) pp. 135-137.

3. M. O. Reade, $A$ radius of univalence for $\int_{0}^{b} e^{-\zeta^{2}} d \xi$, Preliminary report, Bull. Amer. Math. Soc. Abstract 63-3-372.

The Ohio State University and

California Institute of Technology

\title{
FUNCTIONS WHOSE PARTIAL DERIVATIVES ARE MEASURES
}

\author{
BY WENDELL H. FLEMING
}

Communicated by W. S. Massey, July 16, 1958

Let $x$ denote a generic point of euclidean $N$-space $R^{N}(N \geqq 2)$. We consider the space $\mathcal{F}$ of all summable functions $f(x)$ such that the gradient grad $f$ (in the distribution theory sense) is a totally finite measure. $I(f)$ denotes the total variation of the vector measure grad $f$. In case grad $f$ is a function $F$ we have

$$
I(f)=\int_{R^{N}}|F(x)| d x .
$$

We write $H_{k}$ for Hausdorff $k$-measure; and fr $E$ for the frontier of a set $E$. Fr $E$ is rectifiable if it is the Lipschitzian image of a compact subset of $R^{N-1}$.

One ought to be able to determine the primitive $f$ with greater precision than $\operatorname{grad} f$, at least in certain cases. Our main result is that indeed $f$ can be determined up to $H_{N-1}$-measure 0 in two (quite opposed) cases: (1) grad $f$ is a function; (2) the range of $f$ is a discrete set, which we may take to be the integers. More precisely, let $\Im_{1}, \Im_{2}$ be the sets of those $f \in \mathcal{F}$ satisfying (1) and (2) respectively. Let $\mathcal{F}_{01}$ be the set of all Lipschitzian functions $f$ with compact support. Let $F_{02}$ be the set of all functions $f$ with the following property: there exist a closed oriented ( $N-1$ )-polyhedron $A$ and a Lipschitzian mapping $g(w)$ from $A$ into $R^{N}$ such that, for every $x \notin g(A), f(x)$ is the degree of the mapping $g$ at $x$, and $f(x)=0$ for $x \in g(A)$. Write $J(w)$ for the Jacobian vector of $g(w)$, wherever it exists. Let $Q$ denote the set of points $x \in g(A)$ at which there is a nonunique tangent; more precisely, we say that $x \in Q$ if there exist $w, w^{\prime} \in A$ such that: (1) $g$ is 
totally differentiable at $w$ and $w^{\prime} ;(2) g(w)=g\left(w^{\prime}\right)=x ;(3) J(w) \neq 0$, $J\left(w^{\prime}\right) \neq 0$; and (4) $J(w)$ and $J\left(w^{\prime}\right)$ do not point in the same direction.

Definition. A function $f \in \mathcal{F}_{i}$ is precise if there is a sequence $f_{n} \in \mathcal{F}_{0 i} I$-convergent to $f$ such that $\lim _{n} f_{n}(x)=f(x)$ pointwise except in $H_{N-1}$-measure 0 .

THEOREM 1. For $i=1$ or 2 every function $f \in \mathcal{F}_{i}$ is $H_{N^{-}}$almost everywhere equal to a precise function $f^{\prime}$. For $i=1 f^{\prime}$ is uniquely determined $u p$ to $H_{N-1}$-measure 0 . For $i=2 f^{\prime}$ is unique up to $H_{N-1}$-measure 0 if we impose the additional restriction that $f_{n}$ is obtained from a mapping $g_{n}$ as above for which $\lim _{n} H_{N-1}\left(Q_{n}\right)=0$.

The idea of precise function is closely related to Aronszajn's notion of perfect functional completion. In fact:

THEOREM 2. The class of exceptional sets for the perfect functional $I$-completion [1] of the space $\mathcal{F}_{01}$ is the class of all $H_{N-1}$-null sets in $R^{N}$.

Fuglede [6] recently treated the analogous situation when $\operatorname{grad} f \in L^{p}, p>1$. The exceptional sets turn out to be those sets $E$ on which the Riesz potential of appropriate order of some non-negative function in $L^{p}$ can be $+\infty$. Every set of Hausdorff dimension $<N-p$, and none of Hausdorff dimension $>N-p$, is exceptional. For $p=2$, considered previously by Deny and Lions [3], and Aronszajn and Smith [1], the exceptional sets are those of classical outer capacity 0 of order 2 .

A set $E$ has finite perimeter if its characteristic function belongs to $\digamma_{2}$ (see De Giorgi [2]; in [5] I called E Caccioppoli set).

THEOREM 3. Let $E$ have finite perimeter. Then there is a sequence of open sets $E_{n}$ and a set $E^{\prime}$ coincident with $E$ except in a $H_{N}$-null set such that: (1) fr $E_{n}$ is rectifiable for every $n$; and (2) the characteristic function of $E_{n}$ converges to the characteristic function of $E^{\prime}$ in the I-norm and also pointwise except in $H_{N-1}$-measure $0 . E^{\prime}$ is uniquely determined $u p$ to $H_{N-1}$-measure 0 if we require in addition that

$\lim _{n} H_{N-1}\left[x \in \operatorname{fr} E_{n} \mid E_{n}\right.$ does not have an exterior normal ${ }^{1}$ at $\left.x\right]=0$.

Let $E$ be any bounded set in $R^{N}$. Put

$$
\delta(E)=\inf _{f} I(f), f \in F_{01}, f(x) \geqq 1 \text { for } x \in E .
$$

For any set $E$, put

${ }^{1}$ In Federer's sense. 


$$
c(E)=\inf _{\left\{E_{k}\right\}} \sum_{k=1}^{\infty} \delta\left(E_{k}\right), E_{k} \text { bounded, U } E_{k} \supset E .
$$

If we replace $H_{N-1}$ by $c$, then Theorem 2 and the case $i=1$ of Theorem 1 follow easily from [1]. We need to show that

$$
c(E)=0 \text { if and only if } H_{N-1}(E)=0 .
$$

"If" is easy. To prove "only if" we first show that $\delta(E)=\delta_{1}(E)$, where

$$
\delta_{1}(E)=\inf H_{N-1}(\text { fr } \pi), \pi \supset B, \pi \text { polyhedron. }
$$

Then we apply a boxing inequality recently proved by W. Gustin, which states that any polyhedron $\pi$ can be covered by a finite number of cubes $C_{j}$ such that

$$
\sum_{j} H_{N-1}\left(\text { fr } C_{j}\right) \leqq K H_{N-1}(\text { fr } \pi)
$$

where $K$ is a constant depending only on the dimension $N$.

The case $i=2$ and Theorem 3 require in addition results of De Giorgi and Federer, and especially an approximation theorem for closed generalized hypersurfaces a special case of which appears in [4, p. 331].

\section{REFERENCES}

1. N. Aronszajn and K. T. Smith, Functional spaces and functional completion, Ann. Inst. Fourier, Grenoble vol. 6 (1955) pp. 125-186.

2. E. De Giorgi, Su una teoria generale della misura $(r-1)$ dimensionale in uno spazio ad $r$ dimensioni, Ann. Mat. Pura Appl. ser. 4, vol. 36 (1954) pp. 191-213.

3. J. Deny and J. L. Lions, Espaces du type de Beppo Levi, Ann. Inst. Fourier, Grenoble vol. 5 (1954) pp. 305-370.

4. W. H. Fleming and L. C. Young, Generalized surfaces with prescribed elementary boundary, Rend. Circ. Mat. Palermo, ser. 2, vol. 5 (1956) pp. 117-144, 320-340.

5. W. H. Fleming, Functions with generalized gradient and generalized surfaces, Ann. Mat. Pura Appl. ser. 4, vol. 44 (1957) pp. 93-104.

6. B. Fuglede, Extremal length and functional completion, Acta Math. vol. 98 (1957) pp. 171-219.

BROWN UNIVERSITY 